\title{
Enhanced Fourier Shape Descriptor Using Zero-Padding
}

\author{
Iivari Kunttu, Leena Lepistö, and Ari Visa \\ Tampere University of Technology, \\ Institute of Signal Processing, \\ P.O. Box 553, FI-33101 Tampere Finland \\ \{Iivari.Kunttu, Leena.Lepisto, Ari.Visa\}@tut.fi \\ http: //www.tut.fi
}

\begin{abstract}
The shapes occurring in the images are essential features in image classification and retrieval. Due to their compactness and classification accuracy, Fourier-based shape descriptors are popular boundary-based methods for shape description. However, in the case of short boundary functions, the frequency resolution of the Fourier spectrum is low, which yields to inadequate shape description. Therefore, we have applied zero-padding method for the short boundary functions to improve their Fourier-based shape description. In this paper, we show that using this method the Fourier-based shape classification can be significantly improved.
\end{abstract}

\section{Introduction}

The description of the object shape is an important task in image analysis and pattern recognition. The shapes occurring in the images have also a remarkable significance in image classification and retrieval. The basic problem in shape classification is to define similarity between two shapes. Therefore, different visual features (descriptors) have been developed to characterize the shape content of the images. Common shape description techniques have been reviewed in a recent study of Zhang and Lu [17]. Another review of the state of the art in shape description techniques is provided by Loncaric [9].

The shape description techniques can be divided into two types, boundary based and region based techniques [1]. The region based methods consider the whole area of the object whereas the boundary based shape descriptors use only the object boundary in the shape description. The most popularly used region-based methods are different moments [5],[15]. The best-known boundary based shape descriptors include chain codes [3] and Fourier descriptors [13]. Also autoregressive (AR) [2] models have been used in shape description. Simple shape features such as circularity [1], eccentricity, convexity, principle axis ratio, circular variance and elliptic variance [6] include boundary-based descriptors. Recently, growing research interest has been focused on Curvature Scale Space (CSS) shape representation [11] that has been selected to be used as the boundary-based shape descriptor of MPEG-7 standard. 
However, despite the fact that Fourier descriptor is over 30 years old method [4],[13], it is still found to be valid shape description tool. In fact, Fourier descriptor has proved to outperform most other boundary-based methods in terms of classification accuracy and efficiency. This has been verified in several comparisons. Kauppinen et al. [7] made a comparison between autoregressive models and Fourier descriptors in shape classification. In most cases, Fourier descriptors proved to be better in the classification of different shapes. In the comparison made by Mehtre et al. [10], the accuracy of chain codes, Fourier descriptors, and different moments was compared in the shape retrieval. In this case, best results were obtained using moments and Fourier descriptors. In a recent study of Zhang and Lu [16], Fourier descriptors and Zernike moments outperformed CSS representation in terms of retrieval accuracy and efficiency. Similar results were obtained also in [8], in which Fourier descriptors outperformed CSS in defect shape retrieval.

In addition to good classification and retrieval performance, there also other reasons which make Fourier descriptors probably the most popular of the boundarybased shape representations. The main advantages of the Fourier-based shape descriptors are that they are compact and computationally light methods with low dimensionality. Furthermore, they are easy to normalize and their matching is a very simple process. Also their sensitivity to noise is low when only low frequency Fourier coefficients are used as descriptors.

In this paper, the area of Fourier shape descriptors is revisited. We present a method for enhancing the performance of Fourier-based shape description by increasing frequency resolution of the Fourier spectrum calculated for the boundary function of an object shape. Using this method, a more accurate shape representation in frequency domain can be achieved. This is particularly beneficial in the case of objects with relatively short boundary function, in which cases the spectrum estimate has low resolution. The experiments presented in this paper prove that using this technique, the shape classification accuracy can be easily improved.

\section{Shape Representation Using Fourier Descriptors}

In this paper, the shape description methods are based on the boundary line of the object. The boundary can be presented using some shape signature i.e. function derived from the boundary coordinates of the object [1]. Complex coordinate function is a well-known shape signature [7]. It presents the boundary coordinates in an object centered complex coordinate system. Let $\left(x_{k}, y_{k}\right), k=0,1,2, \ldots, N-1$ represent the boundary coordinates, in which $N$ is the length of the boundary. The complex coordinate function $z(k)$ expresses the boundary points in an object centered coordinate system in which $\left(x_{c}, y_{c}\right)$ represents the centroid of the object:

$$
z(k)=\left(x_{k}-x_{c}\right)+j\left(y_{k}-y_{c}\right)
$$

Hence, using this function, the boundary is represented independent of the location of the object in the image. In this way the translation invariance can be achieved. 


\subsection{Fourier Description of the Boundary Function}

Fourier descriptors characterize the object shape in a frequency domain. The descriptors can be formed for the complex-valued boundary function using the discrete Fourier transform (DFT). The Fourier transform of a boundary function generates a set of complex numbers, which characterize the shape in frequency domain. Fourier transform of $z(k)$ is:

$$
F(n)=\frac{1}{N} \sum_{k=0}^{N-1} z(k) e^{-j 2 \pi n k / N}
$$

for $n=0,1,2, \ldots, N-1$. The transform coefficients $F(n)$ form the Fourier spectrum of the boundary function. The translational invariance of this shape representation is based on the object centered shape signature. Furthermore, the coefficients have also to be normalized to achieve invariance to rotation and scaling. The descriptors can be made rotation invariant by ignoring the phase information and using only the magnitudes of the transform coefficients $|F(n)|$. In the case of complex-valued boundary function, the scale can be normalized by dividing the magnitudes of the transform coefficients by $|F(1)|[7]$.

\subsection{Zero-Padding Method}

Even if Fourier descriptor is a powerful tool of boundary-based shape description, its performance is somewhat dependent on the frequency resolution of the Fourier spectrum. When the boundary function $z(k)$ is Fourier transformed, the resulting Fourier spectrum is of the same length as boundary function. Therefore, in the case of short boundary functions the frequency resolution is also low. To obtain better resolution, the number of the datapoints in the boundary function should be increased. In practice, this is not always feasible, because the boundary lines of the objects are usually defined pixel-by-pixel, and the number of the boundary points depends on the image resolution. However, there is an alternative approach for this purpose. Zero-padding [12] is a commonly used method in signal processing. It can be used to increase the frequency resolution by adding zeros to the function to be Fourier transformed. Hence, a new function is defined as:

$$
z_{z p}(k)=\left\{\begin{array}{l}
z(k) \quad \text { for } 0 \leq k \leq N-1 \\
0 \quad \text { for } N \leq k \leq N_{z p}-1
\end{array}\right.
$$

in which $N_{z p}$ is the length of a desired frequency spectrum. By using additional zeros in the input signal of DFT, new spectrum values are being interpolated among the original values in the spectrum. This way, the density of the frequency samples is increased in the spectrum. In practice, the desired spectrum length is selected such that $N_{z p}=2^{p}$ in which $p$ is a positive integer. This is beneficial because DFT is usually 
implemented using FFT algorithm, in which input functions of length $2^{p}$ are preferred to decrease computing time.

\subsection{Descriptors}

The Fourier spectrum represents the frequency content of the boundary function. General shape of the object is represented by the low frequency coefficients of $F(n)$, whereas high frequency coefficients represent the fine details in the object shape. A common approach to shape representation is the use of a subset of low-frequency coefficients as a shape descriptor. Consequently the shape can be effectively represented using a relatively short feature vector. In our experiments, the feature vector is formed using Contour Fourier method [7], which applies the complex coordinate function. In the case of complex valued boundary functions, the coefficients are taken both positive and negative frequency axis. The feature vector is formed as:

$$
x=\left[\frac{\left|F_{-(L / 2-1)}\right|}{\left|F_{1}\right|} \cdots \frac{\left|F_{-1}\right|}{\left|F_{1}\right|} \frac{\left|F_{2}\right|}{\left|F_{1}\right|} \cdots \frac{\left|F_{L / 2}\right|}{\left|F_{1}\right|}\right]^{T}
$$

where $L$ is a constant value that defines the feature vector length (dimensionality).

\section{Experiments}

In this paper, we make experiments to demonstrate the effect of the zero-padding on the shape classification performance. In this experimental part we use a database of industrial defect shapes as a test set.

\subsection{Testing Database}

For testing purposes, we used defect images that were collected from a real industrial process using a paper inspection system [14]. A reason for collecting defect image databases in process industry is a practical need for controlling the quality of production [14]. When retrieving images from a database, the defect shape is one essential property describing the defect class. Therefore, effective methods for the shape representation are necessary. The defects occurring in paper can be for example holes, wrinkles or different kinds of thin or dirt spots. The test set consisted of 1204 paper defects which represented 14 defect classes with each class consisting of 27-103 images. Three example contours of each defect class are presented in figure 1. Within each class, there are defects of different size and orientation. Furthermore, in some classes the boundaries are very varying and sometimes distorted (classes 2, 4 and 10, for example). 


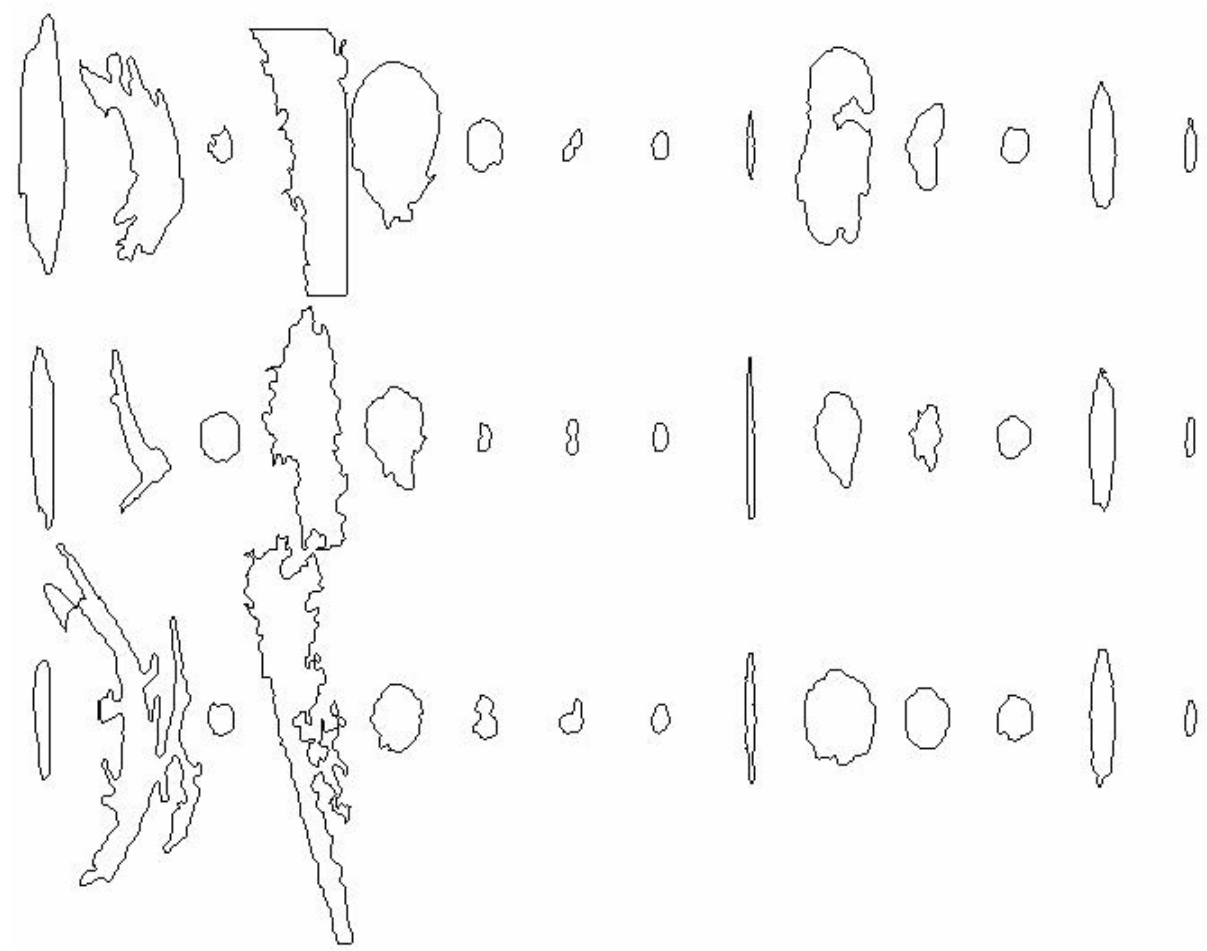

Fig. 1. Three example contours of each 14 paper defect class in the testing database

\subsection{Classification}

The feature vectors for the shapes in the database were calculated for ordinary Contour Fourier descriptors as well as for the same descriptors using zero-padding. The lengths of the boundary lines of the defect shapes were very varying, which can be seen in figure 2. In this figure, the lengths of each 1204 defect boundaries are presented. In our experiments, the boundaries of lower lengths than $N_{z p}$ were inserted with zeros. Two values of $N_{z p}$ were used, namely 512 and 1024 . However, preliminary experiments have showed that in some cases zero-padding also decreases the Fourier-based shape distinction. This can be avoided by emphasizing the zeropadding only to the shortest boundaries in the test set. Therefore, we made an additional experiment, in which only very short boundaries whose length was less than 100 points, were used. These boundaries were zero-padded quite strongly, to 1024 points. The length of the Fourier descriptor $(L)$ was selected to be 16 in all the experiments. It is important to note that the use of zero-padding has no influence on the descriptor dimensionality.

In classification, we used 5-nearest neighbor classifier and leave-one-out validation. The distance metrics was selected to be Euclidean distance, which is a standard 


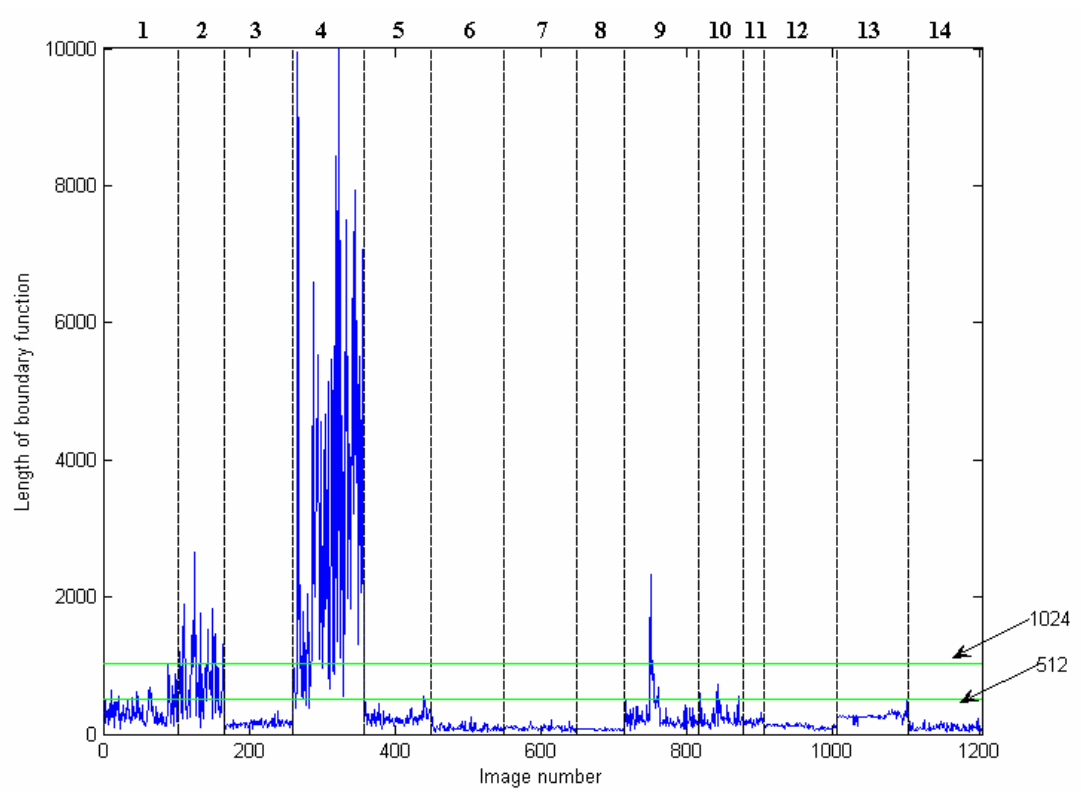

Fig. 2. The lengths of the boundary functions of 1204 paper defects in the testing database. The numbers above the plot correspond to the classes

approach with Fourier descriptors. We carried out the classification using four selected methods, which were ordinary Contour Fourier, Contour Fourier with zeropadding to 512 of 1024 points, and Contour Fourier with zero padding of very short boundaries to 1024 points. Average classification rates of the descriptors are presented for each 14 defect class in figure 3 .

\subsection{Results}

The results presented in figure 3 show that the zero-padding method is able to improve the shape classification rates in most of the defect classes. Particularly, in the classes containing short boundaries the overall result was improved. Therefore, the application of the zero-padding was capable of increasing the average classification performance from $41.2 \%$ to $52.2 \%$. The best average result was achieved using the adaptive zero-padding of very short boundaries to 1024 datapoints. However, all the zero-padding approaches were able to improve the classification results, especially in the classes with short boundaries. For example, in class 11 the classification rate was improved from zero to over $30 \%$.

According to the obtained results, it seems that the most recommendable approach is to use the zero-padding method only to the shortest boundaries, though the other presented approaches produce clear improvement in classification performance as well. 


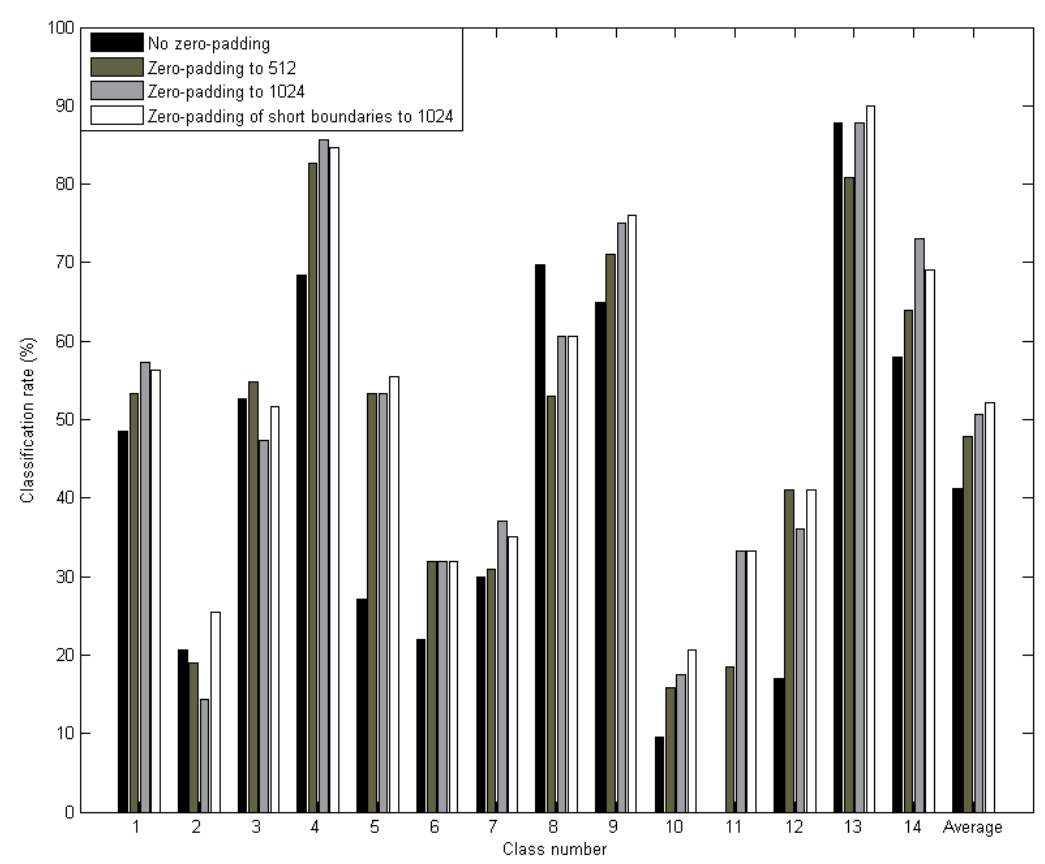

Fig. 3. The average results of 5-NN classification in each 14 defect class

\section{Discussion}

In this paper, a method for improving Fourier-based shape description was presented. The proposed method is a simple and fast tool for improving the shape distinction ability of Fourier descriptors. It is particularly beneficial in the case of short boundary functions, which do not produce adequate frequency resolution to their Fourier spectrum. The zero-padding method as itself is not a novel method, because it has been applied to different kinds of signal processing problems before. However, the new way in which it has been employed to improve shape description has a certain practical value in different shape classification problems.

For experimental purposes, we used industrial defect images, which are quite complicated shape classification task. This is due to the irregularities and variations in the defect shapes. Some of the classes are also somewhat overlapping. It is essential to note that in real defect image classification the shape is not the only classifying feature, because also texture and gray level distribution play a role in defect image description.

The experimental results obtained from this real-world shape classification problem show that using zero-padding the classification performance can be significantly improved. This, on the other hand, does not increase the computational cost, because the dimensionality of the feature vectors remains the same. Zero-padding method 
does not require additional computational capacity in feature extraction, either. This is due to the advanced FFT algorithms [12].

In conclusion, the zero-padding method has proved to an effective tool for enhancing Fourier-based shape description. It is especially effective with short boundary functions of complicated shapes.

\section{Acknowledgment}

The authors wish to thank ABB Oy (Mr. Juhani Rauhamaa) for the paper defect image database used in the experiments.

\section{References}

1. Costa, L.F., Cesar, R.M.: Shape Analysis and Classification, Theory and Practice, CRC Press, Boca Raton, Florida (2001).

2. Dubois, S.R., Glanz, F.H.: An Autoregressive Model Approach to Two-Dimensional Shape Classification, IEEE Transactions on Pattern Analysis and Machine Intelligence Vol. 8 (1986) 55-66

3. Freeman, H., Davis, L.S.: A Corner Finding Algorithm for Chain Coded Curves, IEEE Transactions on Computers Vol. 26 (1977) 297-303

4. Granlund, G.H.: Fourier Preprocessing for Hand Print Character Recognition, IEEE Transactions on Computers Vol. C-21, No. 2 (1972) 195-201

5. Hu, M.K.: Visual Pattern Recognition by Moment Invariants, IRE Transactions on Information Theory Vol. IT-8 (1962) 179-187

6. Iivarinen, J., Visa, A.: An adaptive texture and shape based defect classification, Proceedings of the $14^{\text {th }}$ International Conference on Pattern Recognition, Vol. 1, (1998) $117-122$

7. Kauppinen, H., Seppänen, T., Pietikäinen, M.: An Experimental Comparison of Autoregressive and Fourier-Based Descriptors in 2D Shape Classification, IEEE Transactions on Pattern Analysis and Machine Intelligence, Vol. 17, No. 2 (1995) 201-207

8. Kunttu, I., Lepistö, L., Rauhamaa, J., Visa, A.: Multiscale Fourier Descriptor for ShapeBased Image Retrieval, Proceedings of $17^{\text {th }}$ International Conference on Pattern Recognition, Vol. 2 (2004) 765-768

9. Loncaric, S.: A survey of shape analysis techniques, Pattern Recognition Vol. 31, No. 8 (1998) 983-1001

10. Mehtre, B.M., Kankanhalli, M.S., Lee, W.F.: Shape Measures for Content Based Image Retrieval: A Comparison, Information Processing Management, Vol. 33, No 3, (1997) 319-337

11. Mokhtarian, F., Mackworth, A.K.: Scale-based description and recognition of planar curves and two-dimensional shapes, IEEE Transactions on Pattern Analysis and Machine Intelligence, Vol. 8, No. 1 (1986) 34-43

12. Orfanidas, S.J.: Introduction to Signal Processing, Prentice Hall (1996)

13. Persoon, E., Fu, K.: Shape Discrimination Using Fourier Descriptors, IEEE Transactions on Systems, Man, and Cybernetics, Vol. 7 (1977) 170-179

14. Rauhamaa, J., Reinius, R.: Paper Web Imaging with Advanced Defect Classification, Proceedings of the 2002 TAPPI Technology Summit (2002) 
15. Teague, M.R.: Image Analysis via the General Theory of Moments, Journal of Optical Society of America, Vol. 70, No. 8 (1980) 920-930

16. Zhang, D., Lu, G.: A Comparative Study of Curvature Scale Space and Fourier Descriptors for Shape-Based image Retrieval, Journal of Visual Communication and Image Representation, Vol. 14, No. 1 (2003) 41-60

17. Zhang, D., Lu, G.: Review of shape representation and description techniques. Pattern Recognition, Vol. 37, No. 1, (2004) 1-19 